\title{
Biodegradation of a petroleum-derived groundwater plume reveals the compositional continuum of dissolved organic matter
}

Podgorski D.C. ${ }^{1}$, Zito P. ${ }^{2}$, Smith D.F. ${ }^{2}$, Cao X. ${ }^{3}$, Schmidt-Rohr K. ${ }^{3}$, Wagner S. ${ }^{4}$, Stubbins A. ${ }^{5}$, Cozzarelli I.M. ${ }^{6}$, Bekins B.A. ${ }^{6}$, Spencer R.G.M. ${ }^{2}$

${ }^{1}$ University of New Orleans, New Orleans, USA, dcpodgor@uno.edu

${ }^{2}$ National High Magnetic Field Laboratory, Florida State University, Tallahassee, USA

${ }^{3}$ Brandeis University, Waltham, USA

${ }^{4}$ Rensselaer Polytechnic Institute, Troy, USA

${ }^{5}$ Northeastern University, Boston, USA

${ }^{6}$ United States Geological Survey, Reston and Menlo Park, USA

doi: 10.36291/HIT.2019.podgorski.015

Quantitative and qualitative analytical techniques including dissolved organic carbon (DOC), excitation-emission matrix spectroscopy (EEMS), ultrahigh resolution mass spectrometry (UHR-MS), benzene polycarboxylic acid (BPCA), and nuclear magnetic resonance (NMR) spectroscopy were utilized to measure the molecular-level composition, structural, and reactivity continua of a dissolved organic matter (DOM) plume located at the National Crude Oil Spill Fate and Natural Attenuation Research Site, near Bemidji, Minnesota, USA. All analytical measurements indicate a smooth and continuous change in DOM composition along the centerline of the plume transect. Regardless of methodological selectivity or limitations, results from each technique tracked the same path along the DOM continuum. Moreover, we show that the reactivity of the DOM pool is dependent on the composition observed along the gradient. A detailed understanding of the relationship between the composition and reactivity continua of DOM will aid in our development of miniaturized (lab-on-a-chip) assays that provide quantitative field measurements of DOM reactivity in aquatic ecosystems.

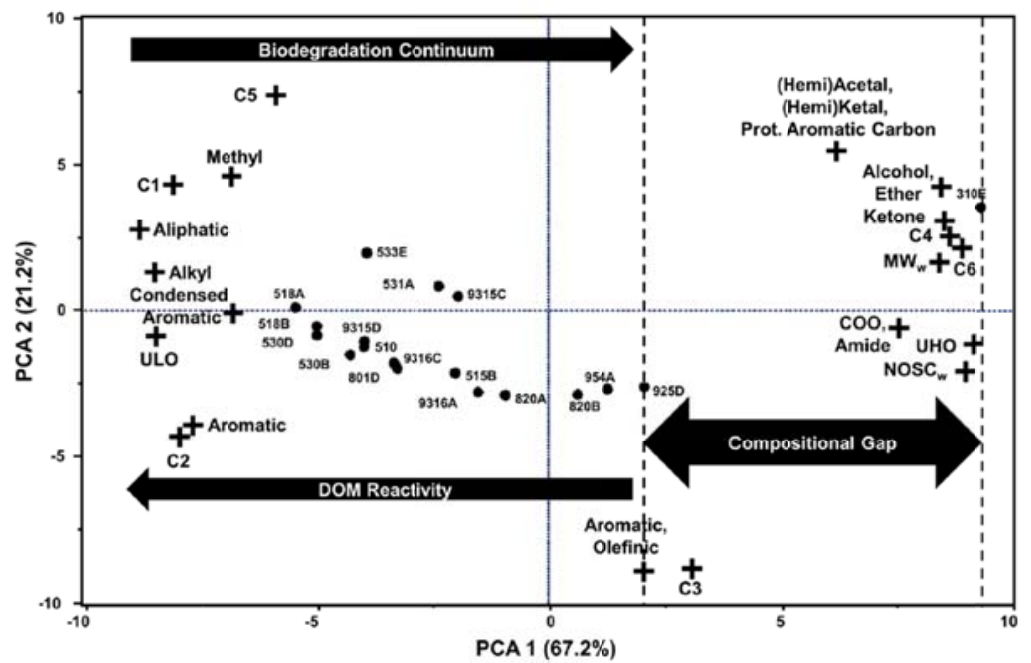

Figure 1. PCA plot of the compositional continuum that results from biodegradation of DOM produced from an aliphatic organic carbon source in a confined aquifer. Changes in fluorescence, molecular-level composition, and structure across the DOM plume transect are smooth and continuous, corresponding with a simultaneous decrease in microbial reactivity. The discontinuity or gap in composition is an indicator of DOM from different source material and corresponding reactivity potential.

Acknowledgements. Research supported by Shell Global Solutions, Enbridge Energy, and U.S. Geological Survey Toxic Substances Hydrology Program. UHR-MS analyses supported by the NHMFL User Program, NSF DMR-1157490. 